\title{
Caracterización histológica y hemodinámica del injerto con isquemia caliente según el método de preservación
}

\author{
Cabello Benavente R, Rodríguez Martínez D*, Hernández Fernández C, del Cañizo López JF*, \\ Lledó García E.
}

\begin{abstract}
Servicio de Urología-Unidad de Preservación Renal Experimental. *Laboratorio de Circulación Artificial. Dpto. Medicina y Cirugía Experimental. Hospital General Universitario Gregorio Marañón. Madrid.
\end{abstract}

Actas Urol Esp. 2008;32(1):75-82

\section{RESUMEN}

CARACTERIZACIÓN HISTOLÓGICA Y HEMODINÁMICA DEL INJERTO CON ISQUEMIA CALIENTE SEGÚN EL MÉTODO DE PRESERVACIÓN

Introducción y Objetivos: Disponer de órganos de donantes a corazón parado abre una vía para solucionar la escasez de órganos para trasplante. Por la naturaleza de estos donantes los riñones sufren un periodo variable de isquemia normotérmica (IN) antes de su extracción, que puede dañar el injerto y condicionar su supervivencia. Minimizar el daño producido al riñón durante la extracción, los diferentes tipos de conservación hipotérmica y su posterior trasplante resulta clave para mejorar los resultados obtenidos con este tipo de injertos.

Pretendemos evaluar mediante un modelo experimental, que somete al injerto renal a un periodo de IN, las consecuencias estructurales y hemodinámicas de la perfusión hipotérmica $(\mathrm{PH})$ frente a la hipotermia simple (HS).

Material y Métodos: Utilizamos 20 mini-pigs. La IN se realizó siempre mediante oclusión del pedículo vascular durante 45 min. Dividimos los órganos extraidos mediante nefrectomia transperitoneal en 4 grupos: $\mathbf{A}$ ( $\mathrm{n}=5$ ), riñones obtenidos tras 45 min. de IN y trasplantados; grupo B $(n=5)$, riñones obtenidos tras IN y trasplantados tras una fase de PH en solución Belzer-gluconato a $4^{\circ} \mathrm{C}$ en nuestro modelo de bomba de vacío no oclusiva. Dos grupos adicionales de órganos fueron trasplantados: sin isquemia normotérmica previa (Ccontrol) o tras IN y 60 min de conservación en HS con Viaspan-UW (D).

Todo el procedimiento fue monitorizado y registrado mediante un sistema informatizado. La resistencia vascular renal (RVR) y el flujo vascular renal (FVR) fueron registrados en tiempo real tras el desclampaje. Llevamos a cabo estudio histológico mediante microscopía convencional y electrónica.

Resultados: Observamos 2 patrones evolutivos post-trasplante:

1. Incremento inicial de RVR con descenso posterior e incremento del flujo vascular: órganos con IN y PH previa al trasplante (grupo B) // órganos sin isquemia previa y trasplante (grupo C-control). Microscopía convencional-electrónica: integridad túbulo-endotelial.

2. Descenso inicial de RVR con incremento posterior: órganos con IN y trasplante directo (A)//órganos con isquemia previa e HS de $60 \mathrm{~min}$. (D). Alteraciones túbulo-endoteliales en micro convencional-electrónica.

Conclusiones: La PH en bomba preserva mejor, a nivel experimental, la ultraestructura túbulo-endotelial de los riñones sometidos a IN prolongada. La hemodinámica post-trasplante de los órganos sometidos isquemia y PH sigue un patrón similar a la de los órganos sin IN previa y trasplantados directamente.

Palabras clave: Isquemia normotérmica. Preservación de órganos.

\section{ABSTRACT}

HYSTOLOGIC AND HEMODYNAMIC ASPECTS OF WARM ISCHEMIC GRAFT IN RELATION TO THE PRESERVATION METHOD

Objective: The non-heart-beating donor has been proposed as a solution to donor shortage for renal transplantation. Because the nature of such donors, the kidneys so derived have been damaged by primary warm ischemia (WI), and so potentially they may never function. Minimizing graft injury is especially important in case of transplantation form marginal donors because of a high rate of delayed graft function or primary nonfunction. The aim of this experimental study is to assess the structural and hemodynamic consequences of hypothermic perfusion (HP) versus cold storage (CS), in renal allograft after a period of WI.

Material and Methods: We used 20 mini-pigs. WI was achieved by vascular pedicle occlusion during 45 min. We divided organs in 4 groups: A $(n=5)$, kidneys with WI and then transplanted; group B $(n=5)$, grafts with WI and implanted after HP with Belzer solution in our computerized perfusion system. Group C-control, $(n=5)$ transplanted without WI and $\mathbf{D}(n=5)$ with WI and 60 min of CS in UW-Viaspan solution.

All the procedure was recorded by a computerized data system. Renal vascular resistance (RVR) and renal vascular flow (RVF) were automatically calculated by means of mathematical formulas after renal transplantation. Subsequently histological study was completed in all cases.

Results: We observed two patterns after transplantation:

1. Initial increase of RVR with posterior decrease and increase of vascular flow: in organs with WI and HP prior to transplantation (group B) // organs transplanted without WI (group C-control). Electronic and conventional microscopy showed integrity of endothelial and tubule structure.

2. Initial decrease with posterior increase of RVR. Organs with WI (group A) // organs with WI and CS (group D). Structural study showed endothelial and tubule disruption.

Conclusión: In our experimental model machine perfusion preserves endothelial and tubule structure of kidneys with WI. After transplantation the hemodynamic pattern of grafts with WI and HP is similar to the control group (without WI and direct transplantation).

Keywords: Warm ischemia. Organ preservation. 
$\mathrm{E}^{1}$ 1 daño provocado al riñón por la isquemia es una causa importante de disfunción del injerto. Después de más de dos décadas de estudio, se sigue trabajando en la comprensión de los efectos de la isquemia y en los mecanismos a través de los cuales lesiona al riñón. La agresión provocada por la extracción, preparación del órgano, preservación, reimplante y reperfusión, puede provocar en las células del injerto necrosis y/o apoptosis ${ }^{1}$, con consecuencias negativas sobre la función y supervivencia del trasplante ${ }^{2}$.

Se considera "donante ideal" aquel entre $16 \mathrm{y}$ 45 años, fallecido como consecuencia de un traumatismo craneoencefálico, generalmente relacionado con un accidente en la carretera ${ }^{3,4}$. No obstante este perfil dista bastante del donante medio actual, de mayor edad y que muere por un evento vascular, ya sea cerebral o cardiaco ${ }^{5,6}$. Con los órganos que se obtienen de éstos se consigue una menor supervivencia del injerto, respecto a la que ofrecen los obtenidos de donantes más jóvenes ${ }^{3}$. De ahí que las investigaciones en preservación renal, se centren en el desarrollo de técnicas que intenten paliar las lesiones celulares que sufre el injerto, consecuencia del proceso de trasplante.

La oferta de riñones para trasplante es limitada, generando una creciente preocupación en busca de alternativas para disponer de un mayor número y mejor calidad de órganos. Por este motivo los donantes a corazón parado (DCP) adquieren día a día más importancia ${ }^{7-9}$. En estudios comparativos, con este tipo de donantes, se consigue una supervivencia del injerto a largo plazo similar a aquellos provenientes de donante vivo o de cadáver con corazón batiente ${ }^{10,11}$.

La perfusión hipotérmica ha sido planteada como un método adecuado para la preservación de riñones con isquemia normotérmica (IN) inicial. Su aplicación en los programas de DCP parece relacionarse con una mejor función inmediata del injerto ${ }^{12}$. Los sistemas de perfusión ofrecen la posibilidad de testar estos órganos "no óptimos" previamente al implante y obtener información hidrodinámica y bioquímica, que permita incluso, aceptar o rechazarlos para el trasplante ${ }^{13}$. La aplicación clínica de determinados algoritmos decisorios debe necesariamente -máxime en el campo del trasplante de órganos- ir precedida de unos sólidos fundamentos experimentales.
Sin embargo, ¿qué datos objetivos, basados en estudios de grandes series randomizadas (perfusión hipotérmica $(\mathrm{PH})$ versus hipotermia simple (HS) nos permiten con un margen suficiente de seguridad aceptar o rechazar órganos? ¿Es positivo que en nuestro país, modelo de donación y trasplante de órganos sólidos para el resto del mundo, se utilicen criterios variopintos de aceptación de riñones según el método de preservación utilizado, la disponibilidad de un determinado dispositivo o la corriente de opinión derivada a una técnica más o menos enraizada?

Quedan abiertos grandes interrogantes pendientes de resolver: ¿Cómo saber cuál es el mejor método de preservación? ¿Puede aplicarse el mismo sistema de conservación de forma universal? ¿Es mejor la PH o la HS? Necesitamos conocer mejor cómo mantienen las dos principales técnicas de conservación la ultraestructura celular en presencia de la agresión isquémica.

\section{OBJETIVOS}

Evaluar mediante un diseño experimental las consecuencias estructurales y hemodinámicas en el trasplante de la $\mathrm{PH}$ frente a la HS como métodos de conservación renal tras un período de IN.

\section{MATERIAL Y MÉTODOS}

Utilizamos 20 mini-pigs (40-45 kg). El manejo de los animales se ha realizado siguiendo la normativa legal según el Real Decreto 223/ 1988, de 14 de Marzo (R. 601): Animales. Protección de los utilizados para experimentación y otros fines científicos (Ministerio de Agricultura, Pesca y Alimentación), que incorpora las disposiciones legales, reglamentarias y administrativas de los estados miembros de la CEE respecto a la protección de los animales utilizados para experimentación y otros fines científicos, de la Directiva del Consejo 86/609/ CEE (Leg. CC. EE. 4390) aprobada el 24 de Noviembre de 1996. Todos los animales fueron hidratados con suero salino fisiológico (1500 ml) y osmofundina (manitol al 20\%, osmolaridad calculada 1.100 mOsm/1, pH 6,5, $250 \mathrm{ml}$ ) desde el inicio de la intervención hasta la extracción de los riñones. Durante todo el procedimiento quirúrgico se realizó perfusión continua en bomba de Heparina al $1 \%$. 
Se procedió en todos los casos a nefrectomía simple por vía transperitoneal. Con el animal en decúbito supino y una vez anestesiado se practicaba una laparotomía media xifopubiana estabilizada con un separador automático de pared. A continuación se exponían con maniobras mínimas de disección el hilio renal correspondiente, disecándose la vena renal sin tracción del pedículo. De la misma forma se procedía con la arteria renal hasta su nacimiento en la aorta para poder obtener el mayor segmento posible de vaso para los implantes posteriores. La IN se realizó siempre mediante oclusión completa (arterial y venosa) del pedículo vascular durante $45 \mathrm{~min}$. Tras esta maniobra se seccionaban los vasos, se procedía a rápida liberación de la grasa perirenal y extracción del riñón, que era inmediatamente sumergido en suero salino frío $\left(4^{\circ} \mathrm{C}\right)$, lavado por gravedad y distribuido al grupo correspondiente.

Dividimos los órganos obtenidos en $\mathbf{4}$ grupos: A $(n=5)$, riñones obtenidos tras $45 \mathrm{~min}$. de IN y trasplantados sin ninguna fase intermedia de preservación hipotérmica; grupo $\mathbf{B}(n=5)$, riñones obtenidos tras IN y trasplantados tras una fase de $\mathrm{PH}$ de $60 \mathrm{~min}$. con solución Belzer-gluconato a $4^{\circ} \mathrm{C}$ en nuestro sistema de perfusión extracorpóreo renal con bomba de vacío no oclusiva controlado por ordenador ${ }^{14}$. Dos grupos adicionales de órganos fueron trasplantados: sin IN previa, C-control, trasplante inmediato, modelo donante vivo $(\mathrm{n}=5)$, o tras IN y $60 \mathrm{~min}$ de $\mathrm{HS}$ mediante inmersión en medio de Viaspan-UW a $4^{\circ} \mathrm{C}$, grupo D $(\mathrm{n}=5)$.

Los implantes fueron llevados a cabo mediante anastomosis termino-lateral de arteria y vena renales a aorta y cava, con suturas no reabsorbibles (polipropileno 6/0). Inmediatamente desde el momento del desclampaje, el flujo vascular renal (FVR) y la presión de perfusión renal (PPR) fueron registrados en tiempo real. Se utilizó un flujómetro ultrasónico T-109 (Transonic Systems Inc), colocado sobre la arteria renal del injerto. La PPR se determinó mediante un transductor desechable (Transpac L978-39 Abbott CCS) ubicado por vía percutánea carotídea hasta la aorta abdominal, próximo al ostium de salida de la arteria. La medida de resistencia vascular renal (RVR) fue realizada por el sistema, según el cociente PPR/FVR ( $\mathrm{mmHg} / \mathrm{ml} / \mathrm{mto}$ ).
El procedimiento fue monitorizado y registrado mediante un sistema informatizado. Todos los órganos fueron sometidos a estudio microscópico convencional y electrónico por el mismo patólogo, que analizó las muestras sin conocer el brazo del estudio al que pertenecían.

\section{RESULTADOS}

Observamos 2 patrones evolutivos post-trasplante (Figs. 1 y 2):

1. Incremento inicial de RVR con descenso posterior. El FVR se mantiene hasta la caída de la RVR. En ese momento emprende un aumento sostenido. Este comportamiento pudo observarse en los órganos con IN y PH previa al trasplante (grupo B) así como en órganos sin isquemia previa y trasplante directo (grupo C-control). Es decir: desde el

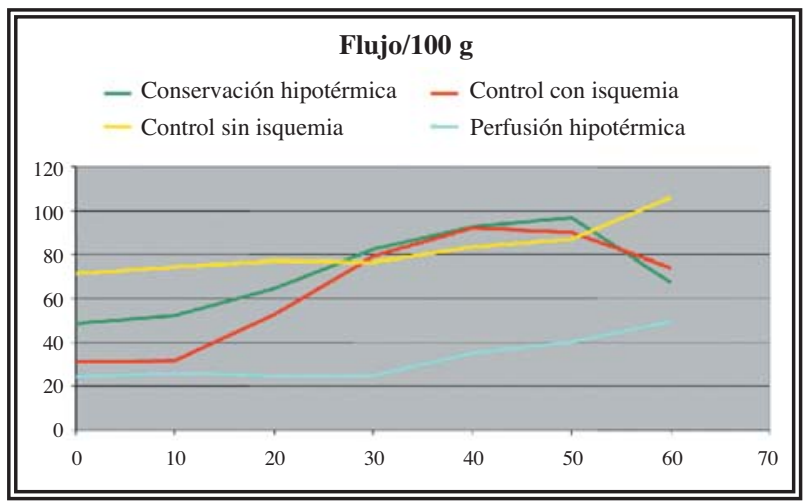

FIGURA 1. Evolución comparativa del Flujo Vascular Renal de los 4 grupos de órganos tras el trasplante. Control con isquemia (grupo A), perfusión hipotérmica (grupo B), control sin isquemia (grupo C), conservación hipotérmica (grupo D).

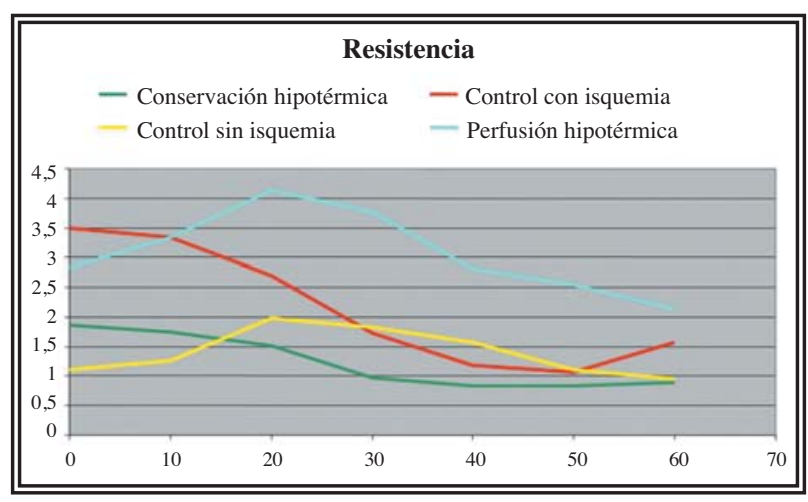

FIGURA 2. Evolución comparativa de la Resistencia Vascular Renal de los 4 grupos de órganos tras el trasplante. Control con isquemia (grupo A), perfusión hipotérmica (grupo B), control sin isquemia (grupo C), conservación hipotérmica (grupo D). 
punto de vista hemodinámico la respuesta de los órganos sometidos a isquemia y perfusión en bomba fue similar al modelo de donante vivo (no isquemia). El estudio microscópico de túbulo y endotelio mostró ausencia de lesiones importantes en la microestructura celular túbulo-endotelial, tanto en el análisis convencional como en el electrónico (Fig. 3).

2. Descenso inicial de RVR, durante los primeros 40 min de reperfusión, con transición gradual a incremento posterior. El FVR aumenta hasta iniciar una progresiva inflexión descendente. Este comportamiento pudo observarse en los órganos con IN y trasplante directo sin preservación (grupo A) así como en órganos con IN, HS y trasplante (grupo D). La respuesta hemodinámica de los órganos sometidos a isquemia e HS por inmersión fue similar al modelo de IN y trasplante directo. El estudio microscópico de túbulo y endotelio mostró edema importante túbular, retracción glomerular y ocupación líquida del espacio de Bowman en el estudio microscópico convencional. En el análisis mediante microscopia electrónica se pudieron observar lesiones endoteliales significativas y ocupación de la luz microvascular por microtrombos de fibrina (Fig. 4).

\section{DISCUSIÓN}

El trasplante es el tratamiento de elección para pacientes con insuficiencia renal crónica terminal. Constituye la opción con relación costo/beneficio más favorable ${ }^{15}$, proporcionando los mejores resultados clínicos con una mejoria significativa de la calidad de vida del enfermo ${ }^{4,16,17}$.
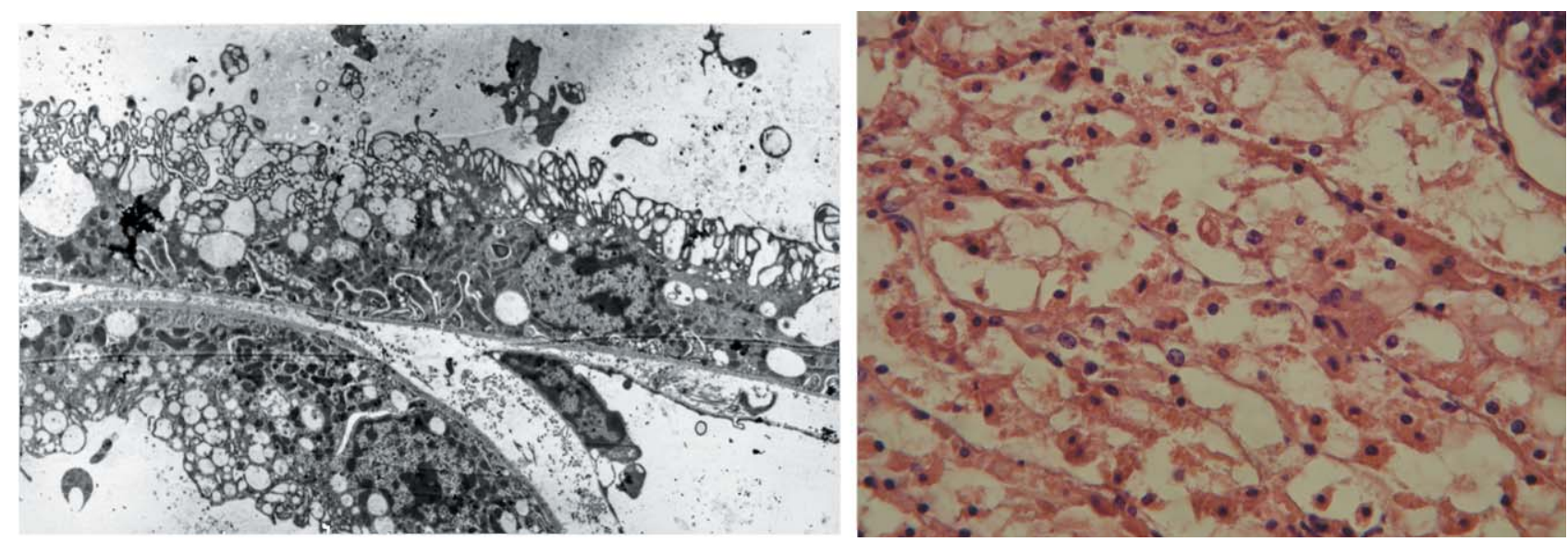

FIGURA 3. Conservación de la estructura túbulo-endotelial en órganos $\sin$ IN (grupo C) y con IN + perfusión en bomba (grupo B). Microscopia electrónica y convencional.
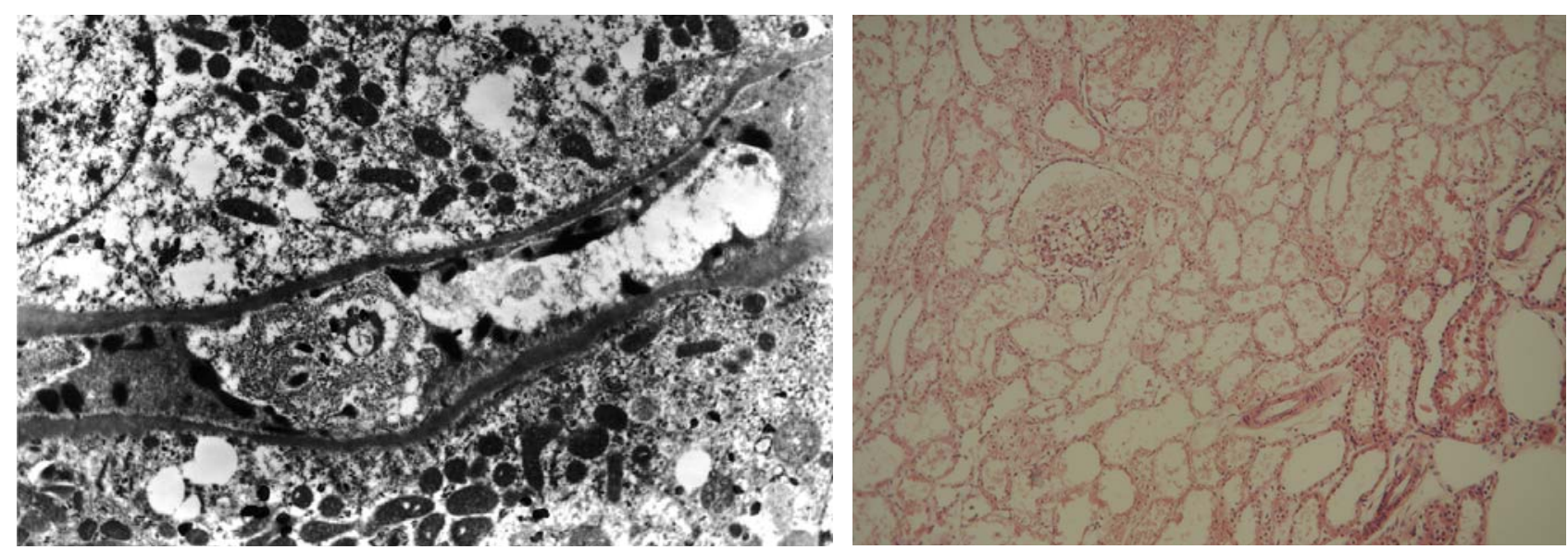

FIGURA 4. Riñones con IN e hipotermia simple (grupo D) o IN sin preservación pre-trasplante (grupo A): lesiones endoteliales, fibrina intraluminal (microscopia electrónica) - edema tubular, retracción glomerular (microscopia convencional). 
Para conservar el órgano donado hasta su implante en el receptor se utilizan técnicas que buscan la supresión de su metabolismo, ya sea mediante inmersión en solución fría o a través de perfusión en máquina. La ventaja fundamental que presenta la conservación hipotérmica es su simplicidad y bajo coste, aunque hay autores que rebaten este argumento al atribuirle un aumento potencial de la estancia hospitalaria y diálisis post-transplante con el consiguiente incremento del costo global ${ }^{18}$. A la agresión que sufre el injerto conservado mediante HS por la propia isquemia hipotérmica hay que sumarle además las asociadas a su extracción (IN) e implante (isquemia caliente y reperfusión). Con la perfusión se pueden paliar algunas de estas agresiones mediante el aporte de sustratos celulares durante la preservación ${ }^{19}$.

Se han utilizado máquinas de perfusión en la conservación de órganos desde hace más de 30 años. Aunque sus ventajas continúan hoy siendo discutidas, sí parece que reducen la incidencia de disfunción precoz del injerto ${ }^{12}$. De las mayores ventajas que ofrecen es la posibilidad de registrar parámetros como la presión de perfusión, el flujo de perfusión o la medición de la concentración de determinados marcadores de lesión tisular para obtener información pronóstica de la viabilidad del órgano ex vivo. Estudios recientes hablan sobre la utilidad de la determinación de la $\alpha$-glutatión-S-transferasa en el líquido de perfusión como marcador de daño celular ${ }^{20-22}$ pero aún no se ha llegado a un marcador de la función renal post-trasplante realmente fiable ${ }^{23}$. Esto nos permitiría seleccionar para su trasplante solamente los órganos con los que previsiblemente se va a alcanzar un éxito clínico. Un hecho inevitable con la perfusión es el incremento de peso del injerto, se produce a consecuencia de la fuga de líquido de perfusión de los capilares provocando edema intersticial ${ }^{24}$. Se considera aceptable un incremento de peso menor al 40\% del órgano. En el momento actual se sabe que la perfusión pulsátil reduce la disfunción tardia del injerto si se compara con la conservación hipotérmica convencional, aunque con los datos disponibles es prematuro generalizar el uso de estos dispositivos ${ }^{12}$. Según los grupos que la realizan, la perfusión podría disminuir los costes de ingreso, al reducir el número de sesiones de diálisis complementarias, y en consecuencia el número de días de ingreso, compensando el mayor gasto que supone este sistema de preservación ${ }^{25}$. Otras ventajas de la $\mathrm{PH}$ es que permite preservar durante más tiempo y en mejores condiciones, el injerto renal. Las posibles desventajas son su elevado coste, el riesgo de avería mecánica del sistema, la posibilidad de lesión endotelial en tiempos prolongados de perfusión y la necesidad de personal técnico especializado durante el proceso ${ }^{13,26}$.

Actualmente no existe ningún método completamente fiable antes del trasplante para evaluar el daño producido en el injerto por la isquémica caliente. Mediante la biopsia, un procedimiento dañino para el injerto o a través de test diseñados para evaluar la resistencia vascular ${ }^{27}$, la producción o fluctuación de determinadas enzimas ${ }^{20,28}$, se ha intentado responder a esta pregunta, aunque desafortunadamente todos estos métodos están entorpecidos por la preservación en hipotermia o por su viabilidad clínica ${ }^{29}$. El daño asociado a la isquemia/reperfusión durante el trasplante es una variable muy importante a tener en cuenta, con consecuencias en la clínica, como el rechazo y la disfunción tardía del injerto. Estas agresiones sobre el injerto provocan una reacción inflamatoria que puede terminar con la fibrosis del parénquima y los vasos ${ }^{30}$. La conservación de la ultraestructura túbulo-glomerular observada en este estudio a nivel experimental asociada a la perfusión hipotérmica (grupo B), tal vez pueda justificar que en estos riñones en la práctica clínica vean reducido en un $20 \%$ el riesgo de disfunción precoz del injerto ${ }^{12}$. La respuesta de los órganos sometidos a IN, tras la perfusión hipotérmica en bomba se asemeja al grupo "ideal", descrito anteriormente (no IN). Esta observación podría ser explicada por una mejor conservación endotelial -a pesar de la agresión isquémica- gracias a la maniobra de perfusión hipotérmica. La similitud hemodinámica correlaciona perfectamente con los hallazgos histológicos, que muestran conservación de la estructura tubulo-endotelial en ambos grupos de órganos.

La perfusión hipotérmica permitiría potencialmente diseñar estudios de viabilidad que descartaran los injertos subóptimos. Esto resultaría muy importante en los programas de órganos de 
DCP. Proporciona la opción de administrar sustratos metabólicos a la célula a través de la solución de perfusión, y la posibilidad de modificar la resistencia vascular, ya sea por la propia perfusión como por maniobras farmacológicas ${ }^{31}$. Los parámetros hemodinámicos que se establecen como punto de corte para rechazar un órgano para su trasplante varian según los autores. Tesi, sitúa el punto de corte en FVR $>70 \mathrm{~mL} / \mathrm{min}$ o una $\mathrm{RVR}<0,4^{32}$. Analizando los datos obtenidos en clínica por Mozes sobre perfusión y su funcionanmiento inmedianto postrasplante, parece que aquellos riñones con un menor FVR $1<100$ $\mathrm{mL} / \mathrm{min})$ y una mayor RVR $(>0,40 \mathrm{mmHg} / \mathrm{mL} /$ min) tienen más riesgo de disfunción inicial ${ }^{33}$. Matsuno rechazaría órganos con un FVR $<0,4$ $\mathrm{ml} / \mathrm{min} / \mathrm{gr}$. y un patrón de RVR con tendencia ascendente $^{31}$. Otros autores consideran como un parámetro favorable y suficiente que la RVR se reduzca durante el tiempo de perfusión ${ }^{24}$. Los valores elevados de RVR obtenidos durante la preservación hipotérmica se han relacionado con la presencia de edema intersticial o microtrombo$\operatorname{sis}^{34}$. Las alteraciones en la hidrodinámica del riñón prefundido pueden ser consecuencia, no sólo de alteraciones morfológicas o estructurales, sino de cambios dinámicos resultado de la sintesis activa de sustancias vasoactivas ${ }^{35}$. Nosotros hemos señalado anteriormente la posibilidad de que pueda existir actividad sintética durante el proceso de perfusión hipotérmica ${ }^{36}$. Sin embargo, en la actualidad se siguen utilizando mayoritariamente otros criterios para aceptar o rechazar órganos, como la edad del donante, la presencia de alteraciones significativas de la histología renal o anatomía del injerto, la positividad de la prueba cruzada entre donante y receptor, así como yatrogenia quirúrgica producida durante las maniobras de extracción ${ }^{33}$.

Comparando dos de los brazos de nuestro estudio que representan el modelo de DCP, según el tipo de preservación posterior al tiempo de isquemia normotérmica, perfusión en bomba (grupo B) o hipotermia simple (grupo D), observamos que en el primer grupo tiene un comportamiento y ultraestructura similares al grupo de trasplante ideal, que es el que representa al de donante vivo (grupo C). Mientras que el segundo grupo se comporta de manera similar al grupo control, que no ha sufrido periodo de preservación tras la isquemia normotérmica (grupo A). La aplicación de una agresión como la isquemia normotérmica, es capaz de alterar la estructura y función del injerto. En trabajos previos de nuestro grupo se demostró a nivel experimental, la capacidad de la perfusión hipotérmica de minimizar el daño estructural producido en el injerto por la isquemia normotérmica. Las alteraciones hemodinámicas) que se observaron (aumento de la RVR) en los riñones sometidos a isquemia normotérmica podian estar relacionados con la vasoconstricción de la arteriola eferente ${ }^{34}$.

Los riñones obtenidos de DCP tienen una mayor tasa de disfunción inicial del injerto, aunque a largo plazo la función del mismo sea equiparable a los obtenidos de donantes a corazón batiente ${ }^{37}$. La formación de radicales libres de oxígeno aumenta la inmunogenidad del injerto facilitando el rechazo del mismo ${ }^{38}$. La pérdida de borde en cepillo es la manifestación más precoz de disfunción del túbulo proximal ante la agresión isquémica ${ }^{39}$. Oliver describió como forma de necrosis tubular, la isquémica o también llamada tubulorrexis ${ }^{40}$. La disfunción mitocondrial está relacionada con la deplección de ATP y acumulación de productos de deshecho, fundamentalmente hipoxantina. Durante la isquemia la xantina deshidrogenasa se transforma en xantina oxidasa, pudiendo ser responsable en parte del daño producido durante la reperfusión ${ }^{41}$. Cuanto más largo es el periodo de isquemia mayores son las lesiones observadas sobre la mitocondria, en forma de edema y desintegración ${ }^{42}$.

Ante las evidencias experimentales y la similitud histológica entre riñones porcinos y huma$\operatorname{nos}^{42}$, se abren nuevos interrogantes que trasladar a la práctica clínica, sobre el método de preservación ideal para aquellos órganos de donantes con isquemia normotérmica (DCP). Los programas clínicos de trasplante renal con utilización de DCP han aumentado la disponibilidad de órganos. Esto ha supuesto un boom en el campo del trasplante renal, proporcionando una fuente de órganos aceptables que ha permitido reducir considerablemente los plazos de espera para pacientes con fallo renal. El futuro es alentador, pero los esfuerzos en investigación no deben cesar hasta lograr aumentar el número, calidad y viabilidad de los trasplantes ${ }^{43}$. Nuestro grupo de 
trabajo tiene grandes expectativas en la conservación mediante perfusión, pues son varias las experiencias que refieren mejores resultados con este tipo de preservación ${ }^{13,44}$. Además, es en los órganos procedentes de donantes en parada cardiaca, en los que la posibilidad de evaluarlos para analizar su viabilidad resulta una herramienta muy útil a la hora de discriminar entre órganos de características potencialmente subóptimas ${ }^{45}$. Durante años el único criterio válido para demostrar la viabilidad de un riñón que ha sido trasplantado era el propio trasplante. Son numerosos los trabajos que hablan sobre la posibilidad que ofrece la perfusión pulsátil para asesorar acerca de la viabilidad del trasplante y proporcionar información de la RVR, el FVR y otros parámetros hemodinámicos ${ }^{46-48}$.

\section{CONCLUSIONES}

La perfusión hipotérmica en bomba preserva mejor, a nivel experimental, la ultraestructura túbulo-endotelial de los riñones sometidos a isquemia normotérmica prolongada. La hemodinámica post-trasplante de los órganos sometidos isquemia y perfusión hipotérmica sigue un patrón similar a la de los órganos sin isquemia normotérmica previa al trasplante e implantados directamente. Los patrones descritos parecen corroborar que en el efecto de la isquemia normotérmica puede atenuarse mediante la perfusión en bomba durante la fase de preservación del injerto previo a su transplante.

Anexo: Proyecto financiado con Beca Investigación FIS 03/1130.

\section{REFERENCIAS}

1. Padanilam BJ. Cell death induced by acute renal injury: a perspectiva of the contributions of apoptosis and necrosis. Am J Physiol Renal Physiol. 2003;284(4): F608-627.

2. Fitzgerald JT, Demos S, Michalopoulou A, Pierce JL, Troppmann C. Assessment of renal ischemia by optical spectroscopy. J Surg Res. 2004;122(1):21-28.

3. Cohen B, D'Amaro J, De Meester J, Persijn GG. Changing patterns in organ donation in Eurotransplant Transplant Int. 1997;10(1):1-6.

4. Cohen B, Smits JM, Haase B, Persijn G, Vanrenterghem Y, Frei U. Expanding the donor pool to increase renal transplantation Nephrol Dial Transplant. 2005;20(1):34-41.

5. Chang GJ, Mahanty HD, Ascher NL, Roberts JP. Expanding the donor pool; can the Spanish model work in the United States?. Am J Transplant. 2003;3(10):1259-1263.

6. Miranda B, Vilardell J, Grinyó JM. Optimizing cadaveric organ procurement: the Catalan and Spanish experience Am J Transplant. 2003;3(10): 1189-1196.
7. Koffman G, Gambado G. Renal transplantation from nonheart-beating donors; a review of the European experience J Nephrol. 2003;16(3):334-341.

8. Nicholson ML. Renal transplantation from non-heart-beating donors. Br J Surg. 1996;83(2):147-148.

9. Kootstra G. The asystolic, or non-heartbeating, donor. Transplantation. 1997;63(7):917-921.

10. Nicholson ML, Metcalfe MS, White SA, Waller JR, Doughman TM, Horsburgh T, et al. A comparison of the results of renal transplantation from non-heart-beating, conventional cadaveric, and living donors. Kidney Int. 2000;58(6):2585-2591.

11. Rengel M, Kanter J, Puerta M, Anaya F, Rodriguez M, Verde E. Kidney transplantation with grafts from non-heart-beating donors. Transplant Proc. 2006;38(3):890-891.

12. Wight JP, Chilcott JB, Holmes MW, Brewer N. Pulsatile machine perfusion vs cold storage of kidneys for transplantation: a rapid and systematic review" Clin Transplant 2003;17(4):293-307.

13. Wight J, Chilcott J, Holmes M, Brewer N. The clinical and cost-effectiveness of pulsatile machine perfusion versus cold storage of kidneys for transplantation retrieved from heart-beating and non-heart-beating donors. Health Technology Assessment. 2003;7(25):1-94.

14. Lledó García E, Hernández Fernández C, Llorente Abarca C, del Cañizo López JF. Computer-controlled isolated kidney hypothermic perfusion system. Actas Urol Esp. 2002; 26(2):77-84.

15. Evans RW, Kitzmann PJ. An economic análisis of kidney transplantation. Surg Clin North Am. 1998;78(1):149-174.

16. Dew MA, Switzer GE, Goycoolea JM, Allen AS, DiMartini A, Kormos RL, et al. Does transplantation produce quality of life benfits?. A quantitative análisis of the literatura. Transplantation. 1997;64(9):1261-1273.

17. Evans RW, Manninen DL, Garrison LP Jr, Hart LG, Blagg CR, Gutman RA, et al. The quality of life of end-stage renal disease patients. N Engl J Med. 1985;312(9):553-559.

18. Matsuno N, Sakurai E, Tamaki I, Uchiyama M, Kozaki K, Kozaki M. The effect of machine perfusion preservation versus cold storage on the function of kidneys from non-heartbeating donors. Transplantation. 1994;57(2):293-294.

19. St Peter SD, Imber CJ and Friend PJ. Liver and kidney preservation by perfusion. Lancet. 2002; 359(9306):604-613.

20. Kievit JK, Nederstigt AP, Oomen AP, Janssen MA, Schoot L, Kootstra G. Release of alpha-glutathione S-transferase (alpha GST) and pi-glutathione S-transferase (pi GST) from ischemic damaged kidneys into the machine perfusaterelevance to viability assessment. Transplant Proc. 1997;29(8):3591-3593.

21. Gok MA, Pelsers M, Glatz JF, Bhatti AA, Shenton BK, Peaston $\mathrm{R}$, et al. Comparison of perfusate activities of glutathione S-transferase, alanine aminopeptidase and fatty acid binding protein in the assessment of non-heart-beating donor kidneys. Ann Clin Biochem. 2003;40(Pt 3):252-258.

22. Daemen JW, Oomen AP, Janssen MA, van de Schoot L, van Kreel BK, Heineman E, et al. Glutathione S-transferase as predictor of functional outcome in transplantation of machine preserved non-heart-beating donor kidney. Transplantation. 1997;63(1):89-93.

23. Gok MA, Pelzers M, Glatz JF, Shenton BK, Buckley PE, Peaston R, et al. Do tissue damage biomarkers used to assess machine-perfused NHBD kidneys predict long-term unction post-transplant?. Clin Chim Acta. 2003;338(1-2): 33-43. 
24. Talbot D, Sentón BK, Buckley PE, et al. Experiences learned in the successful establishment of a nonheart beating donor program for renal transplantation. J Urol. 2003;170 (4 Pt 1):1088-1092.

25. Henry ML. Pulsatile preservation in renal transplantation. Transpl Proc. 1997;29(8):3575-3576.

26. Peerlinck ID, Barlas A, Karameris A, Papalois VE. The effect of machine perfusion on the arteries of porcine kidneys. Exp Clin Transplant. 2005;3(2):375-380.

27. Inman S, Osgood R, Plott W, Richardson B, Lewis R. Identification of kidneys subjected to pre-retrieval warm ischemic injury during hypothermic perfusion preservation. Transplant Proc. 1998;30(5):2309-2310.

28. Kootstra G, Kievit JK, Heineman E. The non heart-beating donor. Br Med Bull. 1997;53(4):844-853.

29. Stubenitsky BM, Booster MH, Nederstigt AP, Kievit JK, Jacobs RW, Kootstra G. Kidney preservation in the next millenium. Transpl Int. 1999;12(2):83-91.

30. Hauet T, Gibelin H, Richer JP, Godart C, Eugene M, Carretier M. Influence of retrieval conditions on renal medulla injury: evaluation by proton NMR spectroscopy in an isolated perfused pig kidney model. J Surg Res. 2000; 93(1):1-8.

31. Matsuno N, Konno O, Mejit A, Jyojima Y, Akashi I, Nakamura Y, et al. Application of machine perfusion preservation as a viability test for marginal kidney graft. Transplantation. 2006;82(11):1425-1428.

32. Tesi RJ, Elkhammas EA, Davies EA, Henry ML, Ferguson RM. Pulsatile kidney perfusion for evaluation of high-risk kidney donors safely expands the donnor pool. Clin Transpl. 1994;8(2 Pt 1):134-138.

33. Mozes MF, Skolek RB, Korf BC. Use of perfusion parameters in predicting outcomes of machine-preserved kidneys. Transplantation Proc. 2005;37(1):350-351.

34. Lledo-Garcia E, Hernandez-Fernandez C, Pedemonte G, Rodriguez-Martinez D, Tejedor-Jorge A, Del Cañizo-Lopez JF. Effects of hypothermic perfusion in isolated pig kidneys after warm ischemia. Transplant Proc. 2007;39(1): 258-260.

35. Knes JM, Hansen TN, Gilligan B, Woo H, Mangino M, Haworth RA. Loss of endothelium-dependent relaxation in abdominal aorta preserved in a co-storage system. Transp Int. 2006;17(11):699-706.

36. Lledó-Garcia E, Hernández-Fernández C, Diez-Cordero JM, García-Barreno P, del Cañizo-López JF. Hydrodynamic and biochemical effects of isolated hypothermic renal perfusion depending on the pump model and perfusion solution. Transplantation Proc. 2003;35(5):1661-1663.

37. Kimber RM, Metcalfe MS, White SA, Nicholson ML. Use of non-heart-beating donors in renal transplantation. 2001; 77(913):681-685.
38. Lu CY, Ar'rajab A, Dawidson I. Ischemia injury during syngenic rat kidney transplantation results in traffcking of inflammatory cells into the kidney and expressions of major histocompatibility and adhesion molecules on renal tubule cells and capillary endothelium J Am Soc Nephrol. 1994; 5: 983.

39. Reimer KA, Ganote CE, Tennings RB. Alterations in renal cortex following ischemic injury. 3. Ultrastructure of proximal tubules after ischemia or autolysis. Lab Invest. 1972;26(4):347-363.

40. Oliver J, Mac Dowell M, Tracy A. The pathogenesis of acute renal failure associated with traumatic and toxic injury, renal ischemia, nephrotoxic damage and the ischemic episode. J Clin Invest. 1951;30(12:1):1307-1439.

41. Gwinner W, Landmesser U, Brandes RP, Kubat B, Plasger $\mathrm{J}$, Eberhard O, et al. Reactive oxygen species and antioxidant defense in puromycin aminonucleoside glomerulopathy. J Am Soc Nephrol. 1997;8(11):1722-1731.

42. Goujon JM, Hauet T, Menet E, Levillain P, Babin P, Carretier M. Histological evaluation of proximal tubule cell injury in isolated perfused pig kidneys exposed to cold ischemia. J Sur Res. 1999;82(2):228-233.

43. Southard JH. Coffee creamer, the bionic man, and organ preservation. Surgery. 2002;131(2):228-229.

44. Booster MH, Wijnen RM, Yin M, Tiebosch AT, Heineman E, Maessen JG, et al. Enhanced resistance to the effects of normothermic ischemia kidneys using pulsatile machine perfusion. Transplant Proc. 1993;25(6):3006-3011.

45. Kozaki K, Sakurai E, Kubota K, Iwamoto H, Hama K, Narumi Y, et al. Prediction of kidney nonfunction alter transplantation with machine perfusion preservation. Transplant Proc. 2000;32(2):275-276.

46. Balupuri S, Strong A, Hoernich N, Snowden C, Mohamed M, Manas D, et al. Machine perfusion for kidneys: how to do it at minimal cost. Transpl Int. 2001;14(2):103-107.

47. Balupuri S, Buckley P, Mohamed M, Cornell C, Mantle D, Kirby $\mathrm{J}$, et al. Assessment of non-heart-beating donor (NHBD) kidneys for viability on machine perfusion. Clin Chem \& Lab Med. 2000;38(11):1103-1106.

48. Polyak MM, Arrington BO, Kapur S, Stubenbord WT, Kinkhabwala M. Calcium ion concentration of machine perfusate predicts early grafts function in expanded criteria donor kidneys. Transpl Int. 1999;12(5):378-382.

Correspondencia autor: Dr. R. Cabello Benavente Servicio de Urología

Hospital General Universitario Gregorio Marañón Dr. Esquerdo 46 - 28007. Madrid

Tel.: 915868000

E-mail autor: ramirocabello@gmail.com Información artículo: Original 